\title{
The Cleaning Coverage Test System of Sweeping Robot
}

\author{
Mao Limin a , Fu Huaming ${ }^{\text {b }}$, Wang Liangyu ${ }^{c}$ \\ School of Electrical Engineering and Automation, Changshu Institute of Technology, Changshu \\ 215500, China

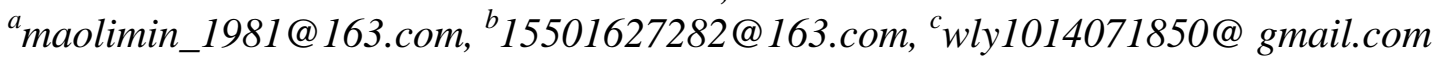

Keywords: LabVIEW; image matching; sweeping robot; coverage;

\begin{abstract}
A cleaning coverage test system based on LabVIEW is presented in this paper, aiming at the uneven cleaning efficiency of sweeping robots on the market. First, the camera is used to capture the test site of the sweeping robot, the resulting image is processed, then the template image is converted into cluster array and the real-time image is matched. The feature is extracted to obtain the position of the sweeping robot, loop to track the path of the sweeping robot, and the coverage of the sweeping robot for this area is calculated.
\end{abstract}

\section{Introduction}

In order to improve the quality of family life, family service robot development is particularly important. The robot has become the focus in the market with its functions of self-cleaning, obstacle avoidance and charging.

Since the 1990s, European and American enterprises have been working on sweeping robots, which have brought various kinds of sweeping robots to the market. At present, sweeping robot has been widely studied and applied in developed countries. IRobot 980 such as the United States, South Korea's Samsung VR10F71UCBC/SC UK Dyson, 360 Eye was welcomed by the market, etc.

In the domestic sweeping robot aspect, more outstanding has the Koworth, its product includes the laser radar, the vision and so on. At present, efficient covering and cleaning in complex indoor environment is still the ultimate goal and challenging technical problem of the robot.

Although there are many kinds of sweeping robots on the market, the cleaning effect is also uneven. Many current sweeping robot coverage tests are done by simulation, and usually have a big difference with real life. In order to facilitate testing the sweeping coverage of robots, in this paper, we build a system to test the coverage rate of sweeping robot in the real environment $[1,2]$.

\section{The coverage test system of cleaning robot}

In this paper, the robot coverage test system is mainly composed of camera and PC. In the range of the camera can shoot, the obtained image will get processed. Transforming template images into clusters and matching real-time images, the position of sweeping robot can be obtained by matching. $[3,4]$ By tracking the path of the sweeping robot, the path is recorded, processed and calculated, and 
the coverage of the sweeping robot is obtained. The system is shown in Fig.1.

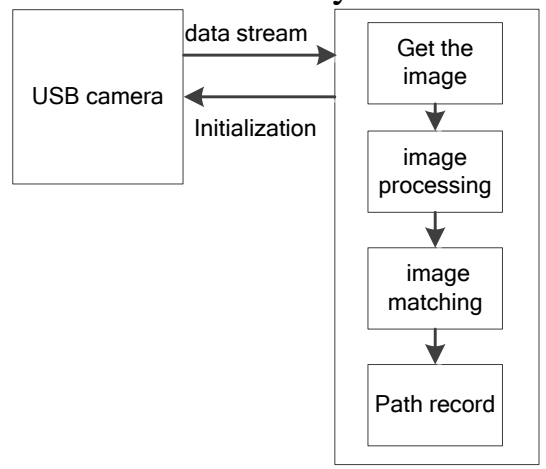

Fig. 1 Test system

3. The upper computer of the coverage test system

\subsection{Image acquisition}

The images collected in this paper are stored in the cache area for subsequent image processing.

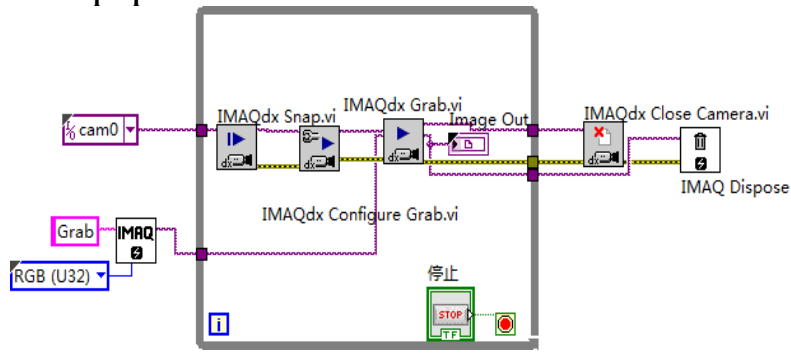

Fig.2 Camera image acquisition

As shown in Fig.2, the image is collected through the USB camera selected by IMAQ, and the result is provided to the IMAQ dx Configure Grab. By configuring the camera, the image is transmitted to the storage area $[5,6]$.

\subsection{Image threshold filtering}

By setting the red or tonal range, range, blue or green or saturation brightness range, choose the required threshold of image filtering, is to be within the scope of all three find corresponding pixel in image Src, VI is used to fill in the target image pixel value, the default value is 1.

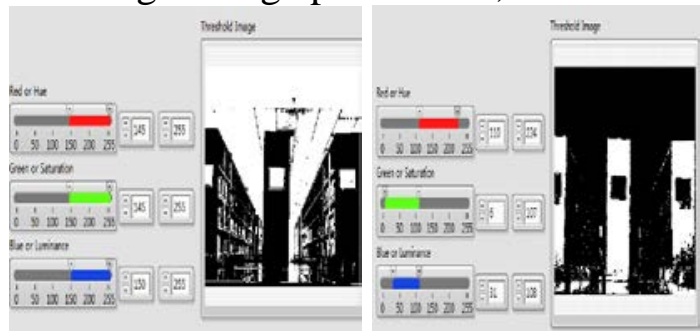

(a)

(b)

Fig.3 Different threshold images

As shown in Figure 3 (a) and (b), the images are obtained from different thresholds, and are 
RGB32 bit pictures. The image needs to be segmented by threshold, the background and noise are filtered out, the filtered part is represented by 0 , and an octal binary image is output in the end $[7,8]$.

\section{3 image matching}

In this paper, the original image is matched according to color and shape, mainly with learning mode and functional mode. The setting of learning mode can be divided into two types :(1) extraction of invariant displacement data; (2) extraction of rotating data. Functional modes can also be divided into three types: (1) only matching shapes, that is, objects have nothing to do with color; (2) only matching colors; (3) matching colors and shapes to make matching more accurate [9].

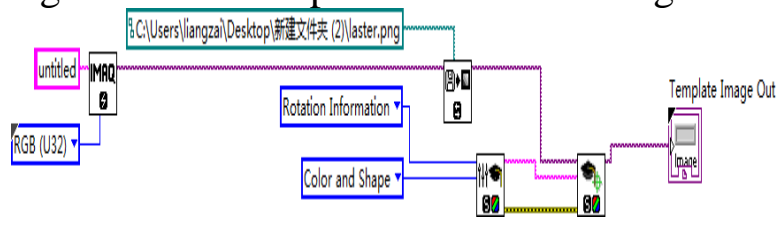

Fig.4 template feature extraction

As shown in Fig.4, template data is extracted and set to IMAQ Match color pattern. Based on color weight fraction (between 0 and 1000), the color mark's contribution to the final color pattern matching score percentage is determined [10].

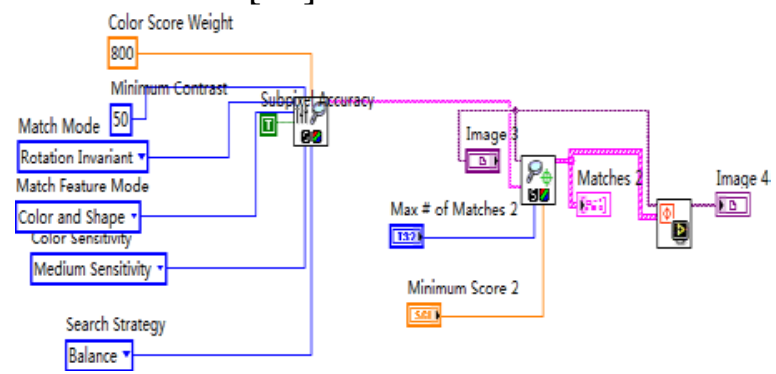

Fig.5 Template matching

\subsection{Template intercept}

When template matching is carried out, the original images of the template through LabVIEW, selects part of the image, extracts the features of the template image, and intercepts the template.

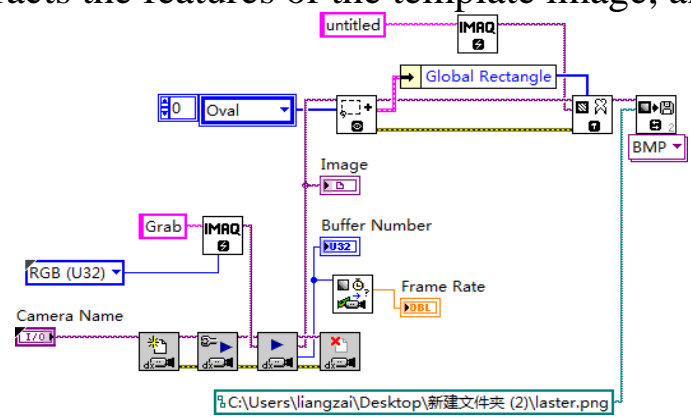

Fig.6 Template interception

\subsection{Effective area calculation}

In this paper, the whole site is photographed, the walking path of the sweeping robot is recorded, and the coverage rate is calculated by the method of circle coverage. When there are barriers in space, 
if does not exclude the obstacles of the area, it will affect the sweeping robot's coverage test. In this paper, a frame of image is intercepted from the site, and the open ground and obstacles are separated as far as possible by binary processing $[11,12]$. Effective area calculation process is shown in Fig.7, and the program is shown in Fig.8.

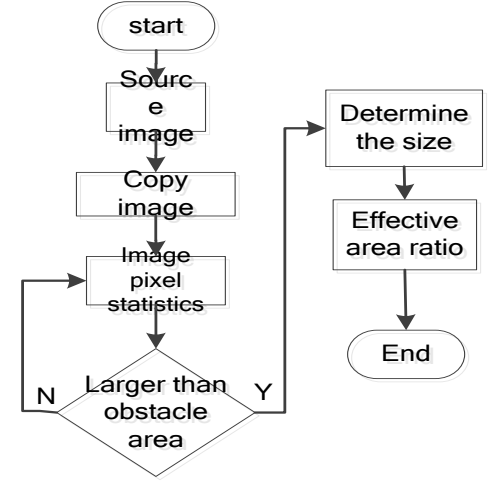

Fig.7 flow of effective area calculation

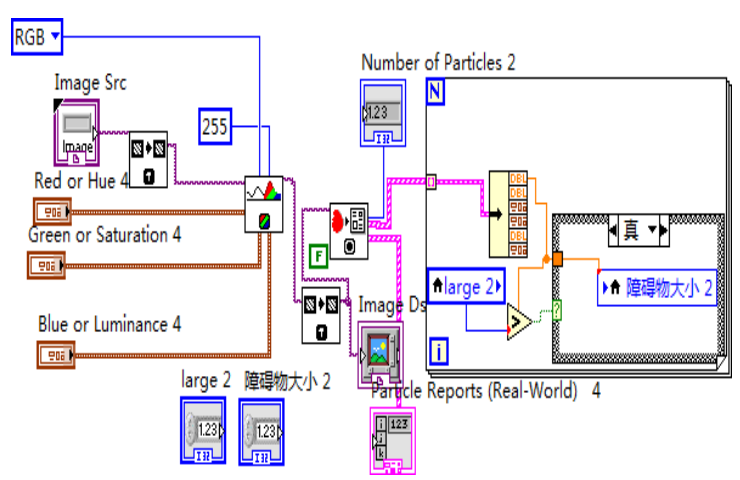

Fig.8 effective area calculation

\subsection{Coverage calculation}

The coordinates within the range of camera acquisition are $(x, y)$ and the value of $(x, y)$ is extracted by unbinding the cluster array. The ellipse covering VI used in this paper is to determine the size and location of an ellipse by defining the intersection of a rectangle tangent to the ellipse. According to the radius of the circle of the robot, draw a circle, the size of which is to record the robot's covering area of the site each frame, such as sweeping robot in $(\mathrm{x} 1, \mathrm{y} 1)$, then use a cluster unbundling and bind to $(\mathrm{x} 1+100,+100 \mathrm{y}),(\mathrm{x} 1-100, \mathrm{y}+100)$, a $(\mathrm{x} 1+100, \mathrm{y}-100),(\mathrm{x} 1-100, \mathrm{y}-100)$, four coordinates of ellipse cluster input cover VI $[13,14]$. Images of the final output finished covering, get RGB images, in order to get the cover, the area of effective for image binary processing, through the particle analysis, return the number of particles detected in the binary image array and contains the most commonly used particle measurement report. According to the name of the cluster decomposition, the images of the particles can be calculated. Compared with the total number of particles in the picture, the coverage rate is obtained [15]. After enlarging the picture, it can be found that it is made up of particles. The size of the image processed in this paper is $480 * 640$, so the total number of particles is 307200 . If the number of particles covered by the area is 100000 , the coverage area is $32 \%$, and the flow chart is shown in Fig.9. The application is shown in Fig.10.
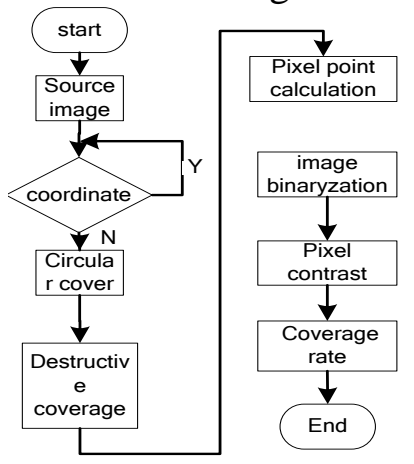

Fig.9 flow chart of coverage calculation

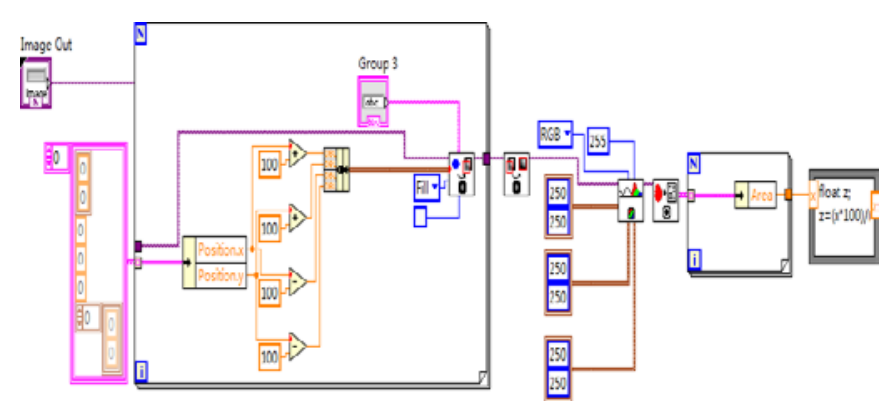

Fig.10 coverage calculation program 


\section{The upper computer of the coverage test system}

\subsection{Template extraction test}

Fig.11 for a camera, a frame images were collected is sweeping the top view of the robot, this article USES the sweeping robot shell reflective cause image cannot be easily identifiable, so use paper cover sweeping robot enclosure, largely overcome the reflective. The sweeping robot template for matching shown in Fig.12.

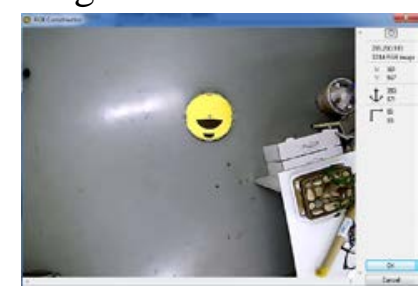

Fig.11 overview of a sweeping robot

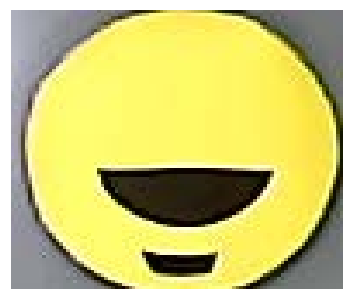

Fig.12 sweeping robot template

\subsection{The matching test}

This article's matching uses the color and shape at the same time to meet the requirements at the same time, which is allowed to rotate 360 degrees. At the beginning of the design we use level match, as shown in Fig13 (a), only when the object rotation does not exceed 4 degrees to match, or the target matching. The rotation matching in this paper allows the deflection range of the object to be 360 degrees. As is shown in Fig.13(b), the test system can still detect the position of the object and its current direction.

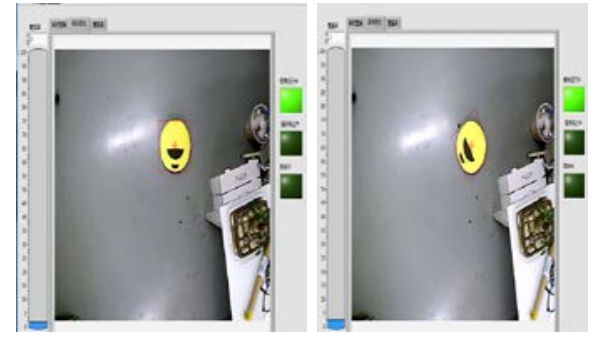

(a) (b)

Fig.13 object shape matching

Due to the different environment, the cause of the light intensity shadow, the color of the same object captured by the camera changes, and the object needs to be 100 percent similar in a perfect match, which is difficult to achieve in practice.

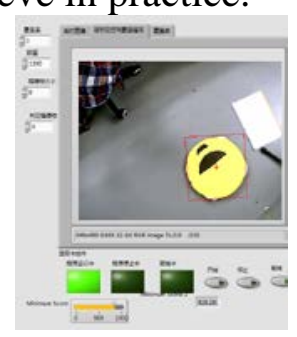

(a)

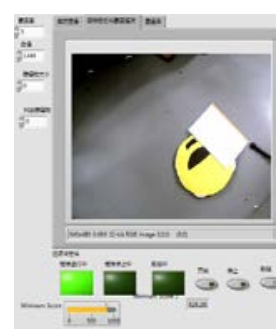

(b)

Fig.14 colour matching of objects

As shown in Fig.14, after the template of graph objects are extracted, as shown in Fig.(a) similarity 
is 828 , object diagram can be seen in the match is very stable without sunscreen. As part of the object is covered, as shown in Fig.(b) is similar to 828 (1000) under the condition of not matching to the location of the object.

By adjusting the similarity, when the similarity is reduced to 700, the position of the object can be matched even if there is an occlusion on the surface of the object, as shown in Fig.15.

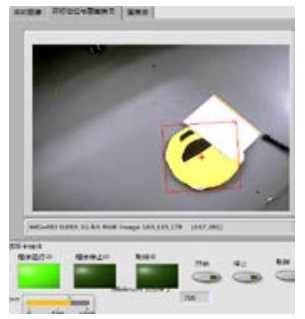

Fig.15 matching graph after adjustment

\subsection{Tracking test}

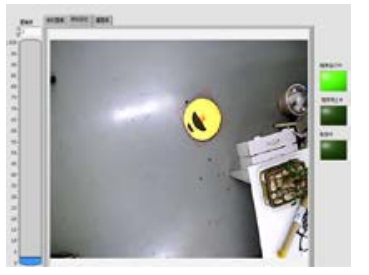

(a)

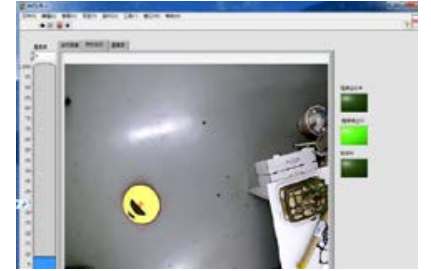

(b)

Fig.16 position before and after motion

As shown in the figure above, when the matching object moves from the position in Fig.16 (a) to the position Fig.16 (b), the system will record the path of the sweeper walking, as shown in Fig.17

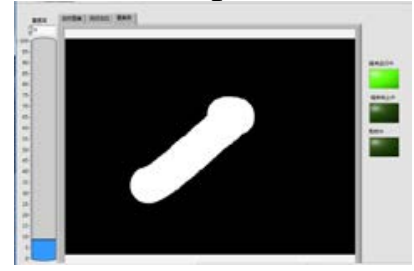

Fig.17 path of system records

\subsection{Effective area test}

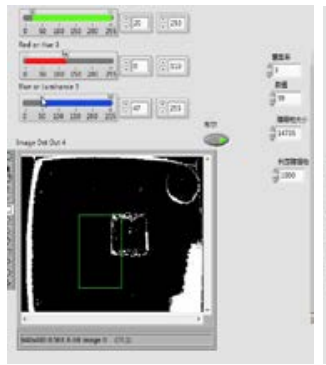

(a)

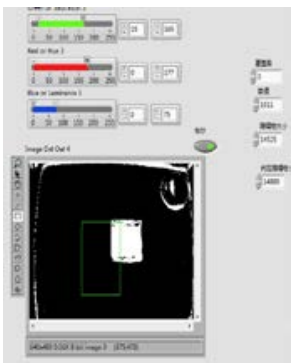

(b)

Fig.18 images displayed with different threshold values

Effective area testing adjusts the saturation of red, green and blue according to the color of the 
obstacle by filtering the threshold value of the image, and replaces it with white pixels when the condition is met. The colour beyond the threshold will be expressed in black, and finally generate two valued images with 8 bits, as shown in Fig18 (a). When the threshold is not chosen properly, only a part of the adjustment can be made, and when the appropriate threshold is adjusted, all the objects are displayed so that the area of the obstacle can be removed in the subsequent coverage calculation. According to the image area calculation module, the graphic area composed of each adjacent pixel on the image can be obtained, as shown in Fig.18 (b). The right upper corner can not be removed simply. Now the determination condition is set to 14000 pixels, if the image is composed of less than this pixel number, it is not an obstacle. As is shown in Fig.18.

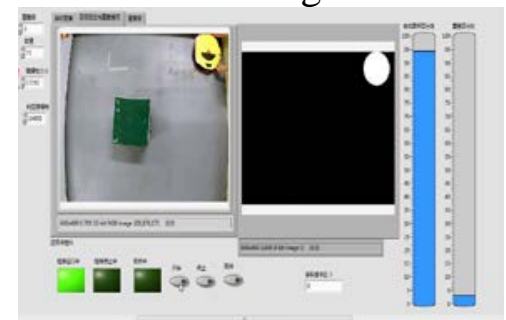

Fig.19 the robot at original point

When sweeping robot begin to work, the ratio of the effective area to the total area is known through the scale bar on both sides, and the ratio of the area swept by the sweeping robot to the effective area is also known, as shown in Fig.19, effective area of $94 \%$ accounted for $6 \%$, the obstacles and sweeping robot in the coverage rate of $3 \%$ when the initial position begin to work.

As shown in Fig.20, the robot carries out two body positions of back-and-forth sweeping when there is no obstacle. At this time, the effective area is considered to be $94 \%$, while the cleaning area becomes $31 \%$.

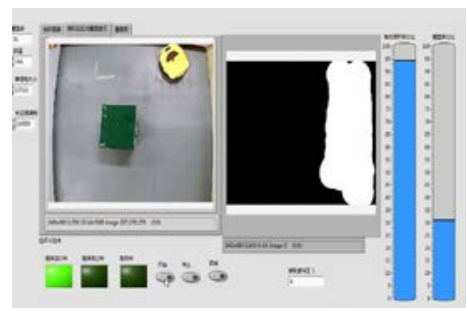

Fig.20 barrier free trajectory of a sweeping robot

When an obstacle occurs, the robot changes its sweeping path as shown in Fig.21. When the sweeper finishes sweeping two positions, it detects obstacles, and the sweeper changes its path. At this point, the effective area is $94 \%$, and the coverage rate is $59 \%$.

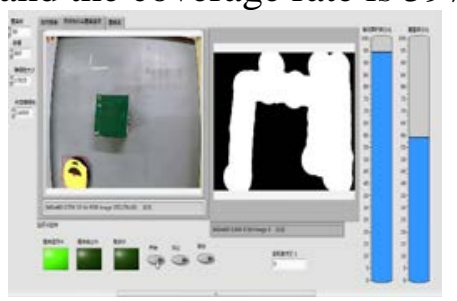

Fig.21 obstacles moving trajectory of a sweeping robot

\section{Conclusions}

In this paper, we use the LabVIEW platform to test the cleaning coverage, using a fixed area monitoring camera to track a more characteristic object in this area. The sweeping coverage system of 
the sweeping robot is realized by covering a certain area of the image through the coordinate position. The sweeping robot tracks the sweeping task in this area and calculates the sweeping robot coverage at the end of the sweeping.

In this paper, some problems still exist, such as camera collection objects which is because of the glare, or sometimes undetectable shadow interference makes the system target, and the camera image acquisition which is due to a slight change in light will lead to a certain fluctuation in the test results of the system. The final results will cause a certain error.

\section{Acknowledgments}

This work was financially supported by Suzhou science and technology project (SYG201504) fund.

\section{References}

[1] GUO Fuqin. The Development and Application of Domestic Service Robot Technology [J]. Science and Technology Innovation, 2012,(24):24-25.

[2] QIU Guangping, CHEN Haitao, YOU Yueling, TAN Hongjian. Intelligent Home Communication System Based on Bluetooth Technology [J] Journal of Chifeng University (NATURAL SCIENCE EDITION), 2016,(07):50-51)

[3] YANG Wenxiang,DU Zhaoping ,ZHUANG Xiaobo. Application of Real-time Image Acquisition and Processing System Based on LabVIEW [J] Electronic design engineering. 2016, (12):159-161+164.

[4] ZHOU Di,XIA Zhelei. Text Image Tilt Correction Method for LabVIEW Platform [J] Journal of China Jiliang University.2012, 32(7):1969-1972.

[5] JIN Yu,LI Hongli. Image Acquisition and Processing Based on LabVIEW and USB Camera [J].Modern electronic technology, 2015, (20):67-70.

[6] GUO Mang,ZHU lianqing, HAN Xiaoquan, LIU Guangyi, FENG Zebin, ZHANG Qingyang. Design of Image Acquisition System Based on LABVIEW for CMOS Camera [J].Automated applications, 2014,(03):15-16+38.

[7] KAN Yuqi, ZHANG Ning, XU Xiping. Design of USB Image Acquisition and Processing System Based on LabVIEW Platform [J]. Industrial Instrumentation and Automation. 2013,(06):80-82

[8] FU Jiajia, LIAO Juan, CHEN Faming. Extraction of machine vision image location information based on LabVIEW[J]2013,(09):62-64.Information and Communication

[9] ZHANG Jin,HUANG Mei,XIA Ling. Medical Image Display and Processing System Based on LabVIEW[J].Computer CD-ROM software and application, 2013,(12):80-81.

[10] LI Qing,YU Wenrong, QIAN Shengqiang. Real-time Image Compression Based on LabVIEW[J].Electronic measurement technology, 2013,(05):7-10+37.

[11] SHEN Jichen,XHANG Wei. Fluorescent Marked Image Detection System Based on LabVIEW [J]. Chemical automation and instrumentation, 2013,(03):309-312.

[12] SONG Bo,MA Fuchang. Digital Image Replacement Experiment Based on Lab VIEW [J].software, 2012, (01):102-105.

[13] DING Sheng,JIANG Xiaoyu,WANG Xi. Research on Image Processing Technology Based on LabVIEW [J]. Microcomputer information, 2010, (29): 204-205.

[14] LIU Chunying,WU Dehua. Research and Application of License Plate Image Recognition Technology Based on LabVIEW[J].Journal of Langfang Teachers College (NATURAL SCIENCE EDITION), 2010,(02):45-47.

[15] WANG Yang, WANG Zhulin. Image Processing Technology Based on Labview[J]Ordnance automation,2009,(01): 89-91 